\title{
Transcriptomic analysis of adaptive mechanisms in response to sudden salinity drop in the mud crab, Scylla paramamosain
}

\author{
Huan Wang ${ }^{1,2}$, Lei Tang ${ }^{1}$, Hongling Wei ${ }^{1}$, Junkai Lu', Changkao Mu² ${ }^{1,2}$ and Chunlin Wang ${ }^{1,2^{*}}$ (D)
}

\begin{abstract}
Background: Scylla paramamosain (Crustacea: Decapoda: Portunidae: Syclla De Hann) is a commercially important mud crab distributed along the coast of southern China and other Indo-Pacific countries (Lin Z, Hao M, Zhu D, et al, Comp Biochem Physiol B Biochem Mol Biol 208-209:29-37, 2017; Walton ME, Vay LL, Lebata JH, et al, Estuar Coast Shelf Sci 66(3-4):493-500, 2006; Wang Z, Sun B, Zhu F, Fish Shellfish Immunol 67:612-9, 2017). While S. paramamosain is a euryhaline species, a sudden drop in salinity induces a negative impact on growth, molting, and reproduction, and may even cause death. The mechanism of osmotic regulation of marine crustaceans has been recently under investigation. However, the mechanism of adapting to a sudden drop in salinity has not been reported.
\end{abstract}

Methods: In this study, transcriptomics analysis was conducted on the gills of S. paramamosain to test its adaptive capabilities over $120 \mathrm{~h}$ with a sudden drop in salinity from $23 \%$ to $3 \%$.

Results: At the level of transcription, 135 DEGs (108 up-regulated and 27 down-regulated) annotated by NCBI non-redundant (nr) protein database were screened. $\mathrm{GO}$ analysis showed that the catalytic activity category showed the most participating genes in the $24 \mathrm{~s}$-tier $\mathrm{GO}$ terms, indicating that intracellular metabolic activities in $\mathrm{S}$. paramamosain were enhanced. Of the 164 mapped KEGG pathways, seven of the top 20 pathways were closely related to regulation of the $\mathrm{Na}^{+} / \mathrm{K}^{+}$-ATPase. Seven additional amino acid metabolism-related pathways were also found, along with other important signaling pathways.

Conclusion: Ion transport and amino acid metabolism were key factors in regulating the salinity adaptation of $S$. paramamosain in addition to several important signaling pathways.

Keywords: Scylla paramamosain, Gill, Osmoregulation, Transcriptional profiling, Differentially expressed gene

\section{Background}

Salinity as a key abiotic parameter that influences the distribution, abundance, physiology, and well-being of crustaceans. [3, 4, 15, 34]. Salinity is also an important factor in the production of crustacean aquaculture [47], which can affect growth, survival, molting, oogenesis, embryogenesis and larval quality $[6,14,21,30,32,35,38]$. The salinity of crustacean aquaculture can easily be affected by a local torrential rain [46]. Fortunately, most marine species which have been studied have

\footnotetext{
* Correspondence: wangchunlin@nbu.edu.cn

${ }^{1}$ School of Marine Science, Ningbo University, Ningbo 315211, Zhejiang, China

${ }^{2}$ Key Laboratory of Applied Marine Biotechnology, Ministry of Education,

Ningbo University, Ningbo 315211, Zhejiang, China
}

osmoregulatory capacities to adjust to the shifting salinities of estuary and wetland waters within limits. In crustaceans, the adaptability of osmoregulation is primarily achieved by the gills $[7,12,16,28,34,36]$. Since regulation of osmotic pressure in marine animals involves energy consumption, drastic changes in salinity can lead to death of the organism.

Recent studies have shown that low salinity influences ion channel activity [33, 37, 44, 45] and L-type free amino acids [1, 25, 39, 42, 43], which are tightly involved with osmoregulation. In particular, the $\mathrm{Na}^{+} / \mathrm{K}^{+}$ -ATPase, a well-known ion channel, is the main ion transport enzyme of post-larvae in crustaceans. Its function in the organism is to enhance adaptability to salinity

(C) The Author(s). 2018 Open Access This article is distributed under the terms of the Creative Commons Attribution 4.0 International License (http://creativecommons.org/licenses/by/4.0/), which permits unrestricted use, distribution, and 
changes through osmoregulation [20, 22, 26, 27]. Chung and Lin [8] cloned the full-length $\alpha$-subunit of the $\mathrm{Na}^{+}$/ $\mathrm{K}^{+}$-ATPase cDNA, indicating the osmoregulatory role of the channel via both mRNA and protein expression. Likewise, Lu et al. [25] completed the cDNA cloning of glutamate dehydrogenase and its expression, indicating that GDH played an important role in controlling osmoregulation through free amino acids in $S$. paramamosain.

Although a great deal of progress had been made in the study of osmotic adjustment in crustaceans, there have been relatively fewer studies on S. paramamosain. In particular, the molecular mechanism of adaptation to sudden salinity drop has not yet been reported. Since $S$. paramamosain is a euryhaline species [40], it is easy to overlook the impact of salinity on the organism's physiology. In the environment, a sudden drop in salinity caused by heavy rain over a short period of time (drop by $>10 \%$ ) may lead to death. In this study, we simulated a drastic reduction in salinity from $23 \%$ o to $3 \%$. Then, the molecular mechanism of adapting to the salinity drop was analyzed by transcriptome analysis.

\section{Methods}

\section{Experimental animals and sectionalization}

A total of 300 randomly selected crabs with a body weight of $\sim 30 \mathrm{~g}$ was selected and kept in a natural water environment with a salinity of $23 \%$ and a temperature of approximately $20{ }^{\circ} \mathrm{C}$. Every 50 crabs were randomly selected (weight $\sim 30 \mathrm{~g}$ ) as a group, with a total of six groups, housed in six cement pools under identical physical and chemical conditions. The salinity of the seawater for three of the groups was adjusted to $3 \%$ from $23 \%$, which dropped by $20 \%$. These three groups were defined as the LS (low salinity) group. The other three groups were defined as the CK groups, where the salinity of seawater was kept at 23\%. All other conditions were the same as the LS group.

It should be noted that the space of a pond in the experiment was big enough for juvenile crabs, and we added a few tiles in ponds as shelter, which could effectively avoid fighting and killing each other.

\section{HE staining}

Gill morphology and ultrastructure of S. paramamosain were observed using light microscopy after hematoxylin (HE) staining. HE staining was conducted according to the method of Wang et al. [41]. First, gills were set in paraffin and sliced into sections with a thickness of up to $5-10 \mu \mathrm{m}$. Then, the sections were de-waxed using $x y-$ lene and rehydrated in an ethanol series. Sections were stained with eosin and HE which purchased from Invitrogen (Carlsbad, CA, USA). Preparation of $4 \%$ paraformaldehyde solution were made using filtered isotonic sea water: the salinity of $23 \%$ and $3 \%$ filtered sea water was used in the $\mathrm{C}$ and LS groups, respectively.

\section{Total RNA isolation and gene expression analysis}

Total RNA was isolated from gill tissue using sqRT-PCR RNAiso Plus (TaKaRa, Dalian, China). The cDNA was synthesized using the Perfect Real Time version of the PrimerScriptTM RT reagent kit with gDNA Eraser (Perfect Real Time) (TaKaRa,) according to manufacturer's instructions. Then, sq-RT-PCR were chosen to analyze genes, and performed in a total reaction volume of $25 \mu \mathrm{l}$ according to the manufacturers' instructions. The $S$. paramamosain beta-actin gene and $18 \mathrm{~S}$ ribosomal RNA gene were selected as the internal control. Primers used in this study are listed in Additional file 1: Table S1.

\section{Transcriptome sequencing}

For Illumina paired-end sequencing, equivalent quantities of total RNA isolated from the three mud crabs were pooled as one sample, and eventually there were three samples in each group, CK and LS. After poly (A) mRNA was purified and fragmented into small pieces, we used random hexamer primers and reverse transcriptase (Invitrogen) to carry out first-strand cDNA synthesis. Second-strand cDNA synthesis was performed with RNase $\mathrm{H}$ (Invitrogen) and DNA polymerase I (New England BioLabs, Beijing, China). A cDNA library was constructed with average insert sizes of 200-500 bp and cDNA sequencing was conducted using the Illumina HiSeqTM 4000 system according to the manufacturer's protocols, with read length of 150 bp Transcriptome Quantification analyses two independent cDNA libraries were constructed for the two organs in parallel according to the Transcriptome protocol. The transcriptome sequencing was performed by BGI (BGI, Shenzhen, China).

\section{Analysis of differentially expressed genes}

Because the annotated genome of $S$. paramamosain has not been published, de novo assembly was used here as reference for further analysis. Firstly, the raw reads were filtered to remove adaptor and low quality sequences. Afterfiltration, clean reads were assembled into unigenes using Trinity de novo assembler, followed by TGICL clustering tool. The reads from control (CK) and experimental group (LS) were mapped against the assembled Unigene using HISAT. The FPKM (fragments per kb per million reads) method was used to calculate the expression abundance. Each unigene was subjected to a BLASTX search against the NCBI non-redundant (nr) protein database with an e-value threshold of 10-3. R package DESeq2 were performed to identify the differentially expressed genes (DEGs). DEG was considered 
as unigene with greater than 2-fold change and $p$-value $<0.05$. Gene ontology (GO) terms and KEGG pathway annotation were achieved using the Blast2GO program and kaas (KEGG Automatic Annotation Server) on-line program (http://www.genome.jp/kaas-bin/kaas_main), respectively.

\section{Results}

Adaptive phenotype of S. paramamosain to sudden drop in salinity

There were four deaths in the CK group within 7 days and 24 deaths in the LS group. The LS death time was

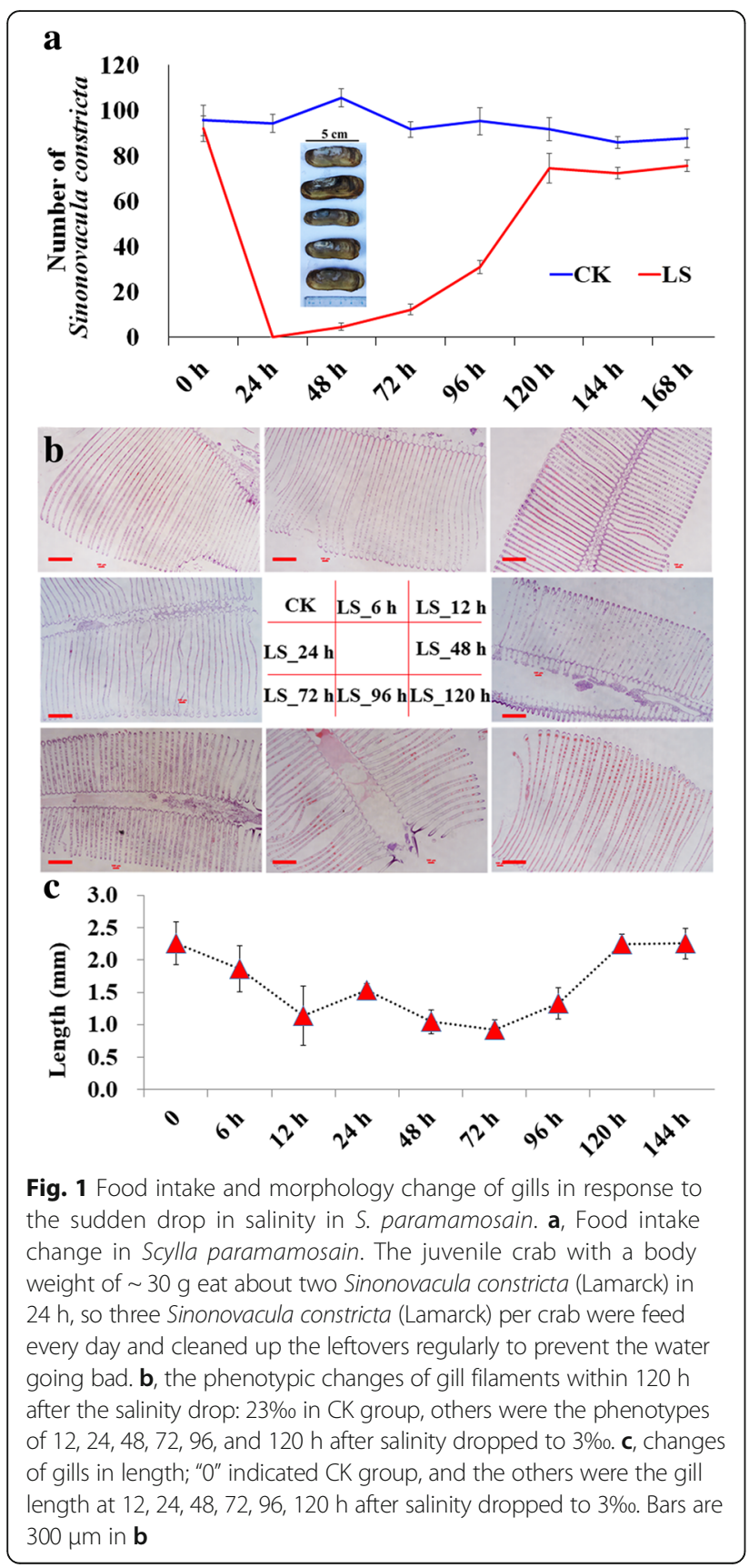

concentrated within 24,48 , and $72 \mathrm{~h}$. In addition, the LS group showed hyperactivity within $48 \mathrm{~h}$. As time went by, the motility was diminished and normalized (Fig. 1a). The LS group did not have food over $72 \mathrm{~h}$, and gradually started to eat over time. Conditions returned to normal after $120 \mathrm{~h}$.

The gills are an important organ in osmoregulation of marine crustaceans, with the gill filament serving as the basic units of function. According to the results of gill slicing, under normal conditions, the gill filaments of the crab in the CK group were regular (Fig. 1b). Gills of crabs in the LS group became shorter and thicker from $6 \mathrm{~h}$ and reached the shortest at $72 \mathrm{~h}$, which was less than half that of gills observed in the CK group (Fig. 1a \& b). Gills then gradually became longer with time and returned to normal after $120 \mathrm{~h}$ (Fig. $1 \mathrm{~b} \& \mathrm{c}$ ). The changes in gill filament anatomy was consistent with those of the physiological activities mentioned above, suggesting that changes in gill filaments play an important regulatory role in adaptation of $S$. paramamosain to decrease in salinity.

\section{Differentially expressed genes (DEGs) in the gill of $S$. paramamosain}

Gills of marine crustaceans play an important role in osmoregulation. In our study, the mud crabs had adjusted to a salinity of $3 \%$ in $120 \mathrm{~h}$ after a sudden drop in salinity. In order to study the molecular mechanism underlying this adaptation, we performed transcriptional profiling at $120 \mathrm{~h}$.

Approximately $39.6 \mathrm{~Gb}$ bases were generated in total on the BGISEQ-500 sequencing platform. Because the genome sequencing of $S$. paramamosain has not yet been elucidated, after reads filtering, Trinity [18] was used to perform de novo assembly with clean reads (Additional file 1: Table S2). Tgicl ([31] Pertea et al., 2003) was used on cluster transcripts to remove abundance and get Unigenes (Additional file 1: Table S3). The proportion of bases with low quality $(<20)$ was very low in all samples, indicating a high quality of sequences. Finally, high-quality transcripts were obtained (Table 1) and used as reference sequences. Genes were annotated using Unigenes by aligning with seven functional database as follows 36,376 (NR: 34.59\%), 38,958 (NT: 37.04\%), 26,425 (Swissprot: 25.13\%), 26,056 (KOG: 24.77\%), 28,890 (KEGG: 27.47\%), 4859(GO: 4.62\%), and 19,756 (InterPro: 18.78\%) (Additional file 1: Figure S2). For functional annotation results, we detected 32,627 CDS by Transdecoder. We also detected 74,041 SSR distributed on 44,075 unigenes, and predicted 12,623 transcription factor (TF) coding unigenes.

A total of 249 genes was differentially expressed in the LS Group and the CK group, including 207 up-regulated genes and 42 down-regulated genes (fold change $>=2.00$ 
Table 1 Quality metrics of transcripts in the gill of Scylla paramamosain

\begin{tabular}{llllllll}
\hline Sample & Total Number & Total Length (bp) & Mean Length & N50 & N70 & N90 & GC(\%) \\
\hline CK_1 & 93,877 & $54,675,298$ & 582 & 952 & 445 & 232 & 45.50 \\
CK_2 & 100,075 & $57,680,722$ & 576 & 938 & 436 & 230 & 45.61 \\
CK_3 & 97,185 & $52,573,783$ & 540 & 803 & 400 & 225 & 45.75 \\
LS_1 & 87,582 & $49,398,653$ & 564 & 885 & 424 & 229 & 45.75 \\
LS_2 & 97,564 & $51,685,899$ & 529 & 777 & 390 & 222 & 45.88 \\
LS_3 & 81,111 & $58,440,218$ & 720 & 1354 & 617 & 262 & 46.89 \\
\hline
\end{tabular}

Sample: Sample name; Total Number: The total number of transcripts; Total Length: The read length of transcripts; Mean Length: The average length of transcripts; N50: The N50 length was used to determine the assembly continuity such that the higher the better. N50 is a weighted median statistic such that $50 \%$ of the total length is contained in the unigenes that are equal to or larger than this value. N70: Similar to the N50; N90: Similar to the N50. GC (\%): the percentage of G and C bases in all transcripts

and adjusted $p$ value $<=0.05$ ) (Fig. 2). Of the 249 DEGs, 217 were annotated at least one of the following: $\mathrm{Nr}$ (192), Nt (160), Swissprot (154), KEGG (163), KOG (152), Interpro (130) and GO (32. No description was found for 3 DEGs (3 of which are down-regulated genes). Of the 192 differential genes annotated in the $\mathrm{Nr}$ database, 57 were described as "hypothetical" in the GenBank database and were also excluded from further analysis. Finally, 135 DEGs that were annotated with $\mathrm{Nr}$ were screened, out of which 108 were up-regulated and 27 were down-regulated genes (Additional file 1: Table S4).

Of these 135 DEGs, BUD13 homolog (ID: CL3353.Con tig2) was the highest-upregulated gene (90.13 fold) followed by serine/threonine-protein kinase (ID: CL90.Contig3, 71.65 fold) and beta-1, 4- $N$-acetylgalactosaminyl transferase (ID: CL2951.Contig2; 67.99 fold) (Additional file 1: Table S4). The three most highly down-regulated genes were uncharacterized proteins LOC107039269 (ID: CL94.Con tig5; - 34.61 fold), kinesin light chain (ID: CL643.Contig10; - 34.03 fold) and aquaporin-12 (ID: CL5376.Contig4; 27.99 fold) (Additional file 1: Table S4). 75 (56\%) showed the highest similarity to genes belonging to phylum Arthropoda (Fig. 3). Of the 75 DEGs, 52 showed highest similarity to genes from class Insecta, and the remainder to genes from class Crustacea (9 DEGs), Merostomata (8 DEGs),
Malacostraca (5 DEGs), and Myriapoda (1 DEG) (Fig. 3). The remaining 60 DEGs (44\%) belonged to the Phylum Chordata (26 DEGs), Protozoa (12 DEGs), Mollusca (10 DEGs), Ciliata (5 DEGs), Nematoda (3 DEGs), Echinodermata (2 DEG), Coelenterata (1 DEG), and Platyhelminthes (1 DEG) (Fig. 3).

\section{Functional annotation}

Gene Ontology (GO) analysis was performed on DEGs using Blast2GO [9, 17]. According to the second-tier GO terms, 32 DEGs were assigned 24 GO annotations which represented three main $\mathrm{GO}$ categories: biological process (18), cellular component (18), and molecular function (23) (Fig. 4 \& Additional file 1: Figure S3). Among them, 24 GO terms were assigned to the up-regulated group and 17 to the down-regulated group. Twelve processes were identified in the biological process category, with 14 DEGs involved in cellular processes, 12 involved in metabolic processes, and 11 involved in single-organism processes. This identified three biological processes as the most strongly affected in the gill of $S$. paramamosain by the sudden drop in salinity (Fig. 4). In the cellular component category, membrane (14), membrane part (10), cell parts (7), and cell (7) were most involved (Fig. 4). In the molecular
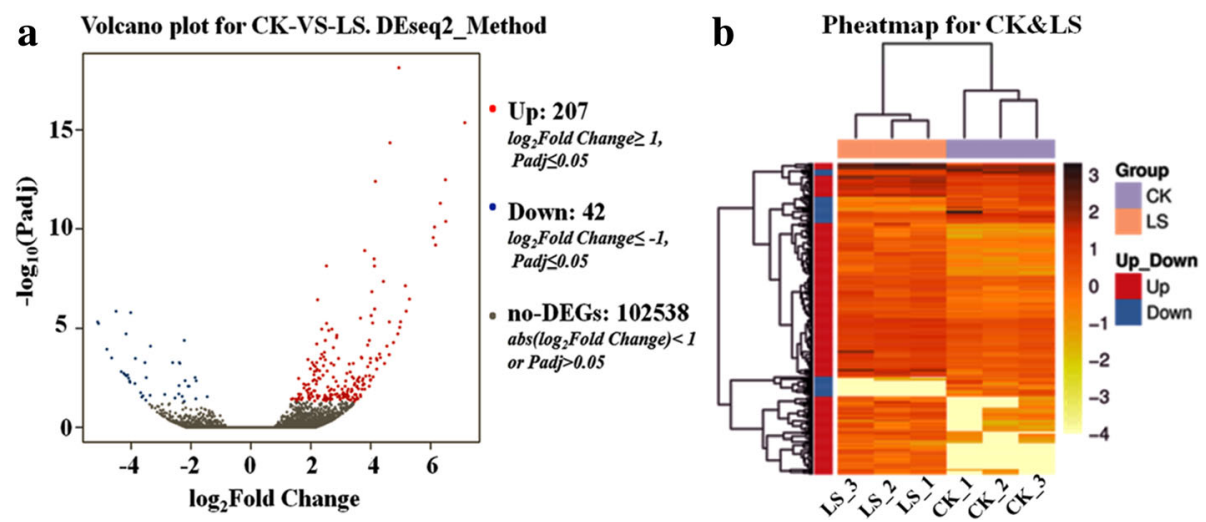

Fig. 2 Differentially expressed genes (DEGs) from the gill of S. paramamosain. $\mathbf{a}$, Volcano plot of DEGs; $\mathbf{b}$, Heatmap of DEGs. Fold change> =2.00 and adjusted $p$ value $<=0.05$ 


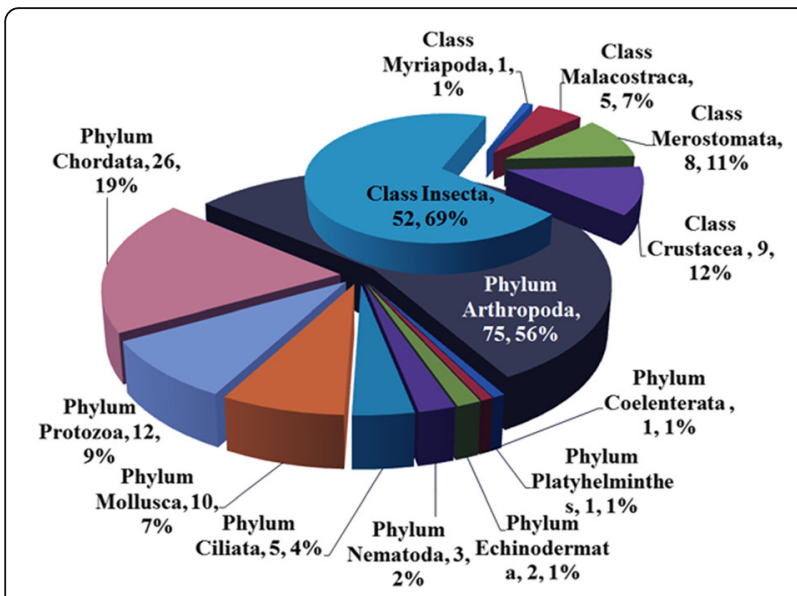

Fig. 3 Class distribution diagram of annotations form $\mathrm{Nr}$ database of 135 differentially expressed genes (DEGs) in response to the sudden drop in salinity in the gill of S. paramamosain. These 135 DEGs showed a certain degree of similarity to genes belonging to phylum Arthropoda (75 DEGs), Chordata (26), Protozoa (12), Mollusca (10), Ciliata (5), Nematoda (3), Echinodermata (2), Coelenterata (1), and Platyhelminthes (1 DEG). The pie chart on the lower side of the image shows taxonomic groups of Phylum, and each fan represented a phylum. The upper pie chart showed taxonomic groups of Class which contain six classes. Numbers and proportion of DEGs are counted in each end of the bar

function category, only four items contained the catalytic activity (16), binding (11), transporter activity (3) and electron carrier activity (1) (Fig. 4). It is worth noting that the catalytic activity category (14) had the most participating genes in the $24 \mathrm{~s}$-tier GO terms. The results

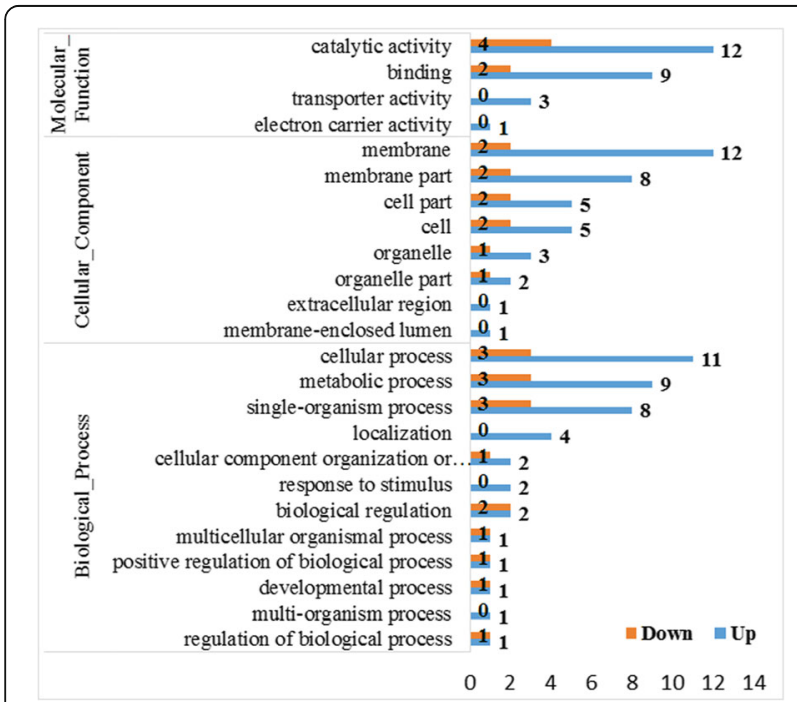

Fig. 4 GO annotation of differentially expressed genes (DEGs) in response to the sudden drop in salinity in the gill of S. paramamosain. DEGs were assigned to second-tier GO categories associated with three parent terms: biological process, cellular component, and molecular function. Blue bars indicate up-regulated DEGs and orange bars indicate down-regulated DEGs. Numbers of DEGs are counted in each end of bar suggested that the salinity of the water environment dropped sharply. In order to maintain life activity, the metabolic activity of the cells in S. paramamosain was strengthened, in particular the catalytic function of enzymes regulating ion changes and osmotic pressure, processes involved in adapting to the new environment with dropped salinity.

In addition, among all the 24 s-tier GO terms, localization, response to stimulus, and multi-organization processes from the biological process category, as well as the extracellular region, membrane-enclosed lumen from cellular component, transporter activity, and electron carrier activity from molecular function categories were all upregulated DEGs with no downregulated DEGs.

\section{DEGs pathway analysis}

With DEGs, KEGG pathway classification was performed (Fig. 5) according to the KEGG database website (http:// www.genome.jp/kegg/pathway.html). Of all DEGs, 119 were mapped in 164 KEGG pathways which were graded into six categories (level 1) according to their biological function, including organismal systems (51), human diseases (47), environmental information processing (40), genetic information processing (28), metabolism (27), cellular processes (22) (Fig. 5a \& b).

Of the 164 pathways, $51(42.86 \%)$ were related to the organismal systems category which was subdivided into nine subsets (level 2): aging, circulatory system, development, digestive system, endocrine system, excretory system, immune system, nervous system, sensory system (Fig. 5a and Table 2). The detailed KEGG pathways of organismal systems and DEGs involved are presented in Table 2. $47(39.50 \%)$ were related to human diseases (Fig. 5a \& b). These genes contained Unigene45614 (toxoplasmosis, ko05145) annotated cytochrome C (Marsupenaeus japonicus) CL979. Contig1 (insulin resistance, ko04931) annotated solute carrier family 2, Unigene 44,862 (Staphylococcus aureus infection, ko05150) annotated keratin, type I cytoskeletal 9 (Marmota marmota marmota), CL979. Contig1 (pathways in cancer, ko05200) annotated solute carrier family 2 , facilitated glucose transporter member 3 (Zootermopsis nevadensis) (Additional file 1: Table S5). It is worth mentioning, these genes were not disease genes, but they were relevant in certain pathologies.

Forty genes (33.61\%) were related to environment information processing which was subdivided into three subsets: signal transduction, signaling molecules and interaction, and membrane transport. Of 164 pathways, 39 genes were most abundant in the signal transduction category, which contained 20 KEGG pathways (Additional file 1: Table S6): AMPK signaling pathway (ko04152), PI3K-Akt signaling pathway (ko04151), HIF-1 signaling pathway (ko04066), cGMP-PKG signaling pathway (ko04022), phosphatidylinositol 


\section{a}

\begin{tabular}{|c|c|c|}
\hline $\begin{array}{c}\text { Organismal Systerms } \\
(51)\end{array}$ & $\begin{array}{r}\text { Endocrine system } \\
\text { Digestive system } \\
\text { Immune system } \\
\text { Aging } \\
\text { Excretory system } \\
\text { Development } \\
\text { Nervous system } \\
\text { Sensory system } \\
\text { Circulatory system }\end{array}$ & 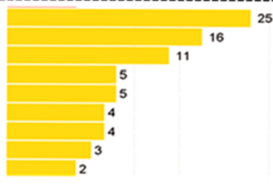 \\
\hline $\begin{array}{c}\text { Human Diseases } \\
\text { (47) }\end{array}$ & $\begin{array}{c}\text { Cancers: Overview } \\
\text { Infectious diseases: Bacterial } \\
\text { Infectious diseases: Parasitic } \\
\text { Cancers: Specific types } \\
\text { Infectious diseases: Viral } \\
\text { Endocrine and metabolic diseases } \\
\text { Neurodegenerative diseases } \\
\text { Antineoplastic resistance } \\
\text { Cardiovascular diseases } \\
\text { Substance dependence }\end{array}$ & 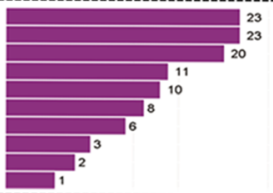 \\
\hline $\begin{array}{c}\text { Environmental Information } \\
\text { Processing (40) }\end{array}$ & $\begin{array}{r}\text { Signal transduction } \\
\text { Signaling molecules and interaction } \\
\text { Membrane transport }\end{array}$ & 1 \\
\hline $\begin{array}{c}\text { Genetic Information } \\
\text { Processing (28) }\end{array}$ & $\begin{array}{r}\text { Folding, sorting and degradation } \\
\text { Translation } \\
\text { Transcription }\end{array}$ & $\overline{\Sigma+\infty}$ \\
\hline $\begin{array}{c}\text { Metabolism } \\
\text { (27) }\end{array}$ & $\begin{array}{r}\text { Global and overview maps } \\
\text { Carbohydrate metabolism } \\
\text { Lipid metabolism } \\
\text { Amino acid metabolism } \\
\text { Energy metabolism } \\
\text { Nesis and metabolism } \\
\text { Glycan biosynthe metabolism } \\
\text { Metabolism of cofactors and vitamins }\end{array}$ & \begin{tabular}{|l|l|} 
& 5 \\
& 4 \\
& 4 \\
$2^{3}$ & \\
\end{tabular} \\
\hline $\begin{array}{c}\text { Cellular Processes } \\
\text { (22) }\end{array}$ & $\begin{array}{r}\text { Cellular community } \\
\text { Transport and catabolism } \\
\text { Cell motility } \\
\text { Cell growth and death }\end{array}$ & $2^{3}$ \\
\hline
\end{tabular}

b

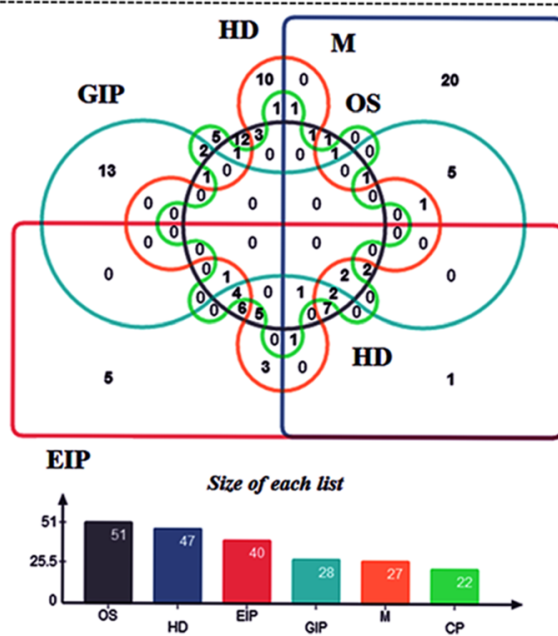

Fig. 5 Pathway classification (a) and differentially expressed genes (DEGs) distribution (b). B, OS: Organismal Systems, HD: Human Disease, EIP: Environmental Information Processing, GIP: Genetic Information Processing, M: Metabolism, CP: Cellular Processes. X axis represents the number of DEGs. Y axis represented functional classification of KEGG. There were seven branches for KEGG pathways: Cellular Processes, Environmental Information Processing, Genetic Information Processing, Human Disease (For animals only), Metabolism, Organismal Systems and Drug Develop (all of the DEGs did not involve)

signaling system (ko04070), hedgehog signaling pathway in fly (ko04341), hedgehog signaling pathway (ko04340), sphingolipid signaling pathway (ko04071), cAMP signaling pathway (ko04024), notch signaling pathway (ko04330), MAPK signaling pathway in fly (ko04013), hippo signaling pathway (ko04390), FoxO signaling pathway (ko04068), MAPK signaling pathway (ko04010), calcium signaling pathway (ko04020), Wnt signaling pathway (ko04310), mTOR signaling pathway (ko04150), hippo signaling pathway in fly (ko04391), Ras signaling pathway (ko04014), and phospholipase D signaling pathway (ko04072). In addition, the other pathways of environment information processing were involved, such as ABC transporters (ko02010) which belonged to membrane transport, ECM-receptor interaction (ko04512), cell adhesion molecules (CAMs) (ko04514), and neuroactive ligand-receptor interaction (ko04080), which belonged to signaling molecules and interaction. Throughout all of environment information processing pathways, the results indicated gill as an important organ for salinity regulation for S. paramamosain. After the gills detected the salinity drop, the salinity drop transformed into stress signals transmitted to other parts of the body, thereby initiating regulation mechanism used by the gills. A complex 
Table 2 Organismal Systems pathways and DEGs involved

\begin{tabular}{|c|c|c|c|}
\hline Pathway & DEGs genes (51) & Pathway ID & Level 2 \\
\hline Longevity regulating pathway - multiple species & Unigene32292, Unigene35344, Unigene35501 & ko04213 & Aging \\
\hline Longevity regulating pathway & Unigene32292, Unigene35501, CL3443.Contig1 & ko04211 & Aging \\
\hline Longevity regulating pathway - worm & CL3933.Contig2 & ko04212 & Aging \\
\hline Cardiac muscle contraction & Unigene11331 & ko04260 & Circulatory system \\
\hline Adrenergic signaling in cardiomyocytes & Unigene11331, Unigene44161 & ko04261 & Circulatory system \\
\hline Vascular smooth muscle contraction & Unigene44161 & ko04270 & Circulatory system \\
\hline Osteoclast differentiation & Unigene19465, Unigene31660 & ko04380 & Development \\
\hline Axon guidance & Unigene40814 & ko04360 & Development \\
\hline Dorso-ventral axis formation & Unigene34154 & ko04320 & Development \\
\hline Protein digestion and absorption & $\begin{array}{l}\text { Unigene12705, CL6154.Contig2, Unigene38622, } \\
\text { CL922.Contig5, Unigene11331, CL922.Contig9, } \\
\text { CL452.Contig3, CL922.Contig7, Unigene17070, } \\
\text { CL3933.Contig2 }\end{array}$ & ko04974 & Digestive system \\
\hline Bile secretion & $\begin{array}{l}\text { Unigene12705, Unigene33785, Unigene38622, } \\
\text { Unigene11331, CL979.Contig3, CL979.Contig1, } \\
\text { Unigene17070 }\end{array}$ & ko04976 & Digestive system \\
\hline Mineral absorption & $\begin{array}{l}\text { Unigene12705, Unigene15443, Unigene38622, } \\
\text { Unigene11331, Unigene17070 }\end{array}$ & ko04978 & Digestive system \\
\hline Carbohydrate digestion and absorption & Unigene11331 & ko04973 & Digestive system \\
\hline Vitamin digestion and absorption & CL4600.Contig3 & ko04977 & Digestive system \\
\hline Salivary secretion & Unigene10578, Unigene11331 & ko04970 & Digestive system \\
\hline Gastric acid secretion & Unigene11331 & ko04971 & Digestive system \\
\hline Pancreatic secretion & Unigene11331 & ko04972 & Digestive system \\
\hline Thyroid hormone signaling pathway & $\begin{array}{l}\text { Unigene33785, Unigene111, Unigene113, } \\
\text { Unigene41655, Unigene36212, Unigene31721, } \\
\text { Unigene11331, Unigene112, CL979.Contig3, } \\
\text { CL979.Contig1, Unigene17270 }\end{array}$ & ko04919 & Endocrine system \\
\hline Adipocytokine signaling pathway & $\begin{array}{l}\text { Unigene33785, CL3443.Contig1, CL979.Contig3, } \\
\text { CL979.Contig1 }\end{array}$ & ko04920 & Endocrine system \\
\hline Insulin secretion & $\begin{array}{l}\text { Unigene33785, Unigene11331, CL979.Contig3, } \\
\text { CL979.Contig1, CL5287.Contig1 }\end{array}$ & ko04911 & Endocrine system \\
\hline Thyroid hormone synthesis & $\begin{array}{l}\text { Unigene10157, Unigene27634, Unigene11857, } \\
\text { Unigene11331 }\end{array}$ & ko04918 & Endocrine system \\
\hline PPAR signaling pathway & CL1683.Contig3, CL1683.Contig5, CL2638.Contig2 & ko03320 & Endocrine system \\
\hline Estrogen signaling pathway & Unigene10157, Unigene35344, Unigene27634 & ko04915 & Endocrine system \\
\hline Progesterone-mediated oocyte maturation & Unigene10157, Unigene27634 & ko04914 & Endocrine system \\
\hline Glucagon signaling pathway & Unigene33785, CL979.Contig3, CL979.Contig1 & ko04922 & Endocrine system \\
\hline Renin-angiotensin system & Unigene49236 & ko04614 & Endocrine system \\
\hline Insulin signaling pathway & Unigene32292, Unigene35501, Unigene44161 & ko04910 & Endocrine system \\
\hline Oxytocin signaling pathway & Unigene44161 & ko04921 & Endocrine system \\
\hline GnRH signaling pathway & CL6599.Contig1 & ko04912 & Endocrine system \\
\hline Proximal tubule bicarbonate reclamation & $\begin{array}{l}\text { Unigene12705, Unigene15693, Unigene38622, } \\
\text { Unigene11331, Unigene17070 }\end{array}$ & ko04964 & Excretory system \\
\hline Aldosterone-regulated sodium reabsorption & Unigene11331 & ko04960 & Excretory system \\
\hline $\begin{array}{l}\text { Endocrine and other factor-regulated calcium } \\
\text { reabsorption }\end{array}$ & Unigene11331 & ko04961 & Excretory system \\
\hline Antigen processing and presentation & $\begin{array}{l}\text { Unigene10157, Unigene35344, Unigene27634, } \\
\text { Unigene1 1857, Unigene41750 }\end{array}$ & ko04612 & Immune system \\
\hline NOD-like receptor signaling pathway & Unigene10157, Unigene27634 & ko04621 & Immune system \\
\hline
\end{tabular}


Table 2 Organismal Systems pathways and DEGs involved (Continued)

\begin{tabular}{|c|c|c|c|}
\hline Pathway & DEGs genes (51) & Pathway ID & Level 2 \\
\hline Hematopoietic cell lineage & Unigene15456, Unigene47891l, Unigene15457 & ko04640 & Immune system \\
\hline B cell receptor signaling pathway & Unigene15456, Unigene47891, Unigene15457 & ko04662 & Immune system \\
\hline Platelet activation & Unigene44161 & ko04611 & Immune system \\
\hline Fc gamma R-mediated phagocytosis & CL6599.Contig1, CL4852.Contig1 & ko04666 & Immune system \\
\hline Long-term potentiation & Unigene44161 & ko04720 & Nervous system \\
\hline GABAergic synapse & CL3399.Contig2 & ko04727 & Nervous system \\
\hline Dopaminergic synapse & Unigene44161 & ko04728 & Nervous system \\
\hline Glutamatergic synapse & CL6599.Contig1, Unigene5252, CL3399.Contig2 & ko04724 & Nervous system \\
\hline Phototransduction & CL6217.Contig3, CL41.Contig2 & ko04744 & Sensory system \\
\hline Olfactory transduction & CL6217.Contig3, CL41.Contig2 & ko04740 & Sensory system \\
\hline Inflammatory mediator regulation of TRP channels & Unigene44161 & ko04750 & Sensory system \\
\hline
\end{tabular}

molecular signal feedback mechanism for regulation could ultimately achieve osmotic pressure balance of as an adaptive response to a sudden salinity drop.

Functional enrichment was also performed on DEGs according to the above KEGG pathway classification. The top 20 pathways (Fig. 6a \& Table 3) showed that seven pathways were directly related to the active regulation of the $\mathrm{Na}^{+} / \mathrm{K}^{+}$-ATPase enzyme: Proximal tubule bicarbonate reclamation (ko04964), protein digestion and absorption (ko04974), bile secretion (ko04976), thyroid hormone signaling pathway (ko04919), mineral absorption (ko04978), insulin secretion (ko04911), thyroid hormone synthesis (ko0491), and the regulatory genes of $\mathrm{Na}^{+} /$ $\mathrm{K}^{+}$-ATPase were all up-regulated。Crustacean $\mathrm{Na}^{+}$/ $\mathrm{K}^{+}$-ATPase plays an important role in the regulation of hematopoietic osmotic pressure at different salinities [2]. Current research on crustacean $\mathrm{Na}^{+} / \mathrm{K}^{+}$-ATPase has been extensively reported $[10,23]$ suggesting that it is a widespread P-type ATPase that plays an important role in maintaining $\mathrm{Na}^{+}, \mathrm{K}^{+}$homeostasis [11]. As all known, free amino acids $[1,25,39,42,43]$ also played an important role in osmoregulation. In addition, several pathways connected with amino acid metabolism were detected, such as for arginine biosynthesis

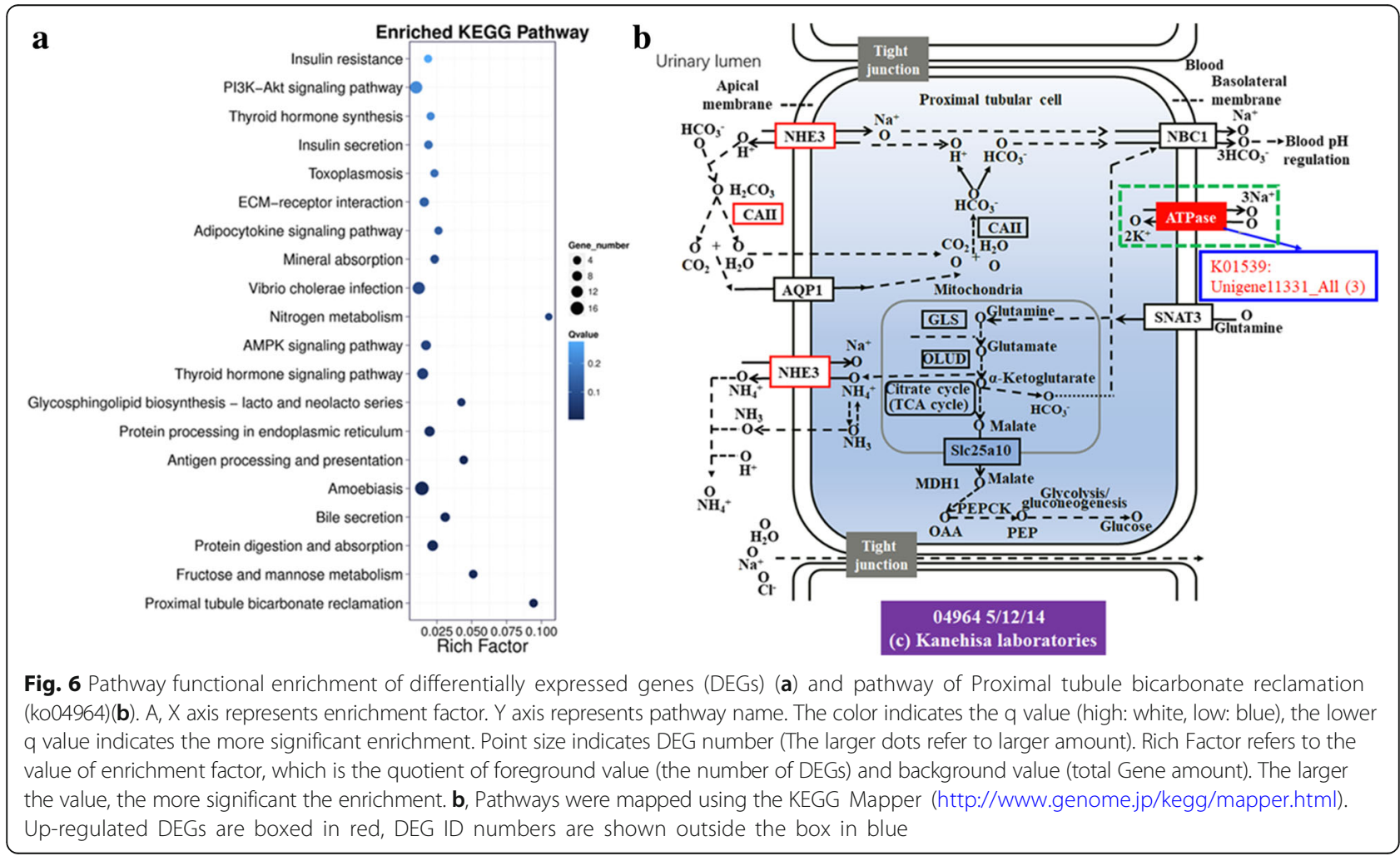


Table 3 The greatest functional classification differences between CK and LS

\begin{tabular}{|c|c|c|c|c|}
\hline $\mathrm{NO}$ & Pathway & $\begin{array}{l}\text { DEGs genes (119) / All genes } \\
\text { (28890) with pathway annotation }\end{array}$ & $P$ value & Pathway ID \\
\hline 1 & Proximal tubule bicarbonate reclamation & $4(4.2 \%) / 53(0.18 \%)$ & $1.239607 \mathrm{e}-05$ & ko04964 \\
\hline 2 & Fructose and mannose metabolism & $5(4.2 \%) / 98(0.34 \%)$ & 0.000239203 & ko00051 \\
\hline 3 & Protein digestion and absorption & $10(8.4 \%) / 456(1.58 \%)$ & 0.000280083 & ko04974 \\
\hline 4 & Bile secretion & $7(5.9 \%) / 226(0.78 \%)$ & 0.0003098698 & ko04976 \\
\hline 5 & Amoebiasis & 17 (14.3\%) / 1195 (4.14\%) & 0.0004429313 & ko05146 \\
\hline 6 & Antigen processing and presentation & $5(4.2 \%) / 113(0.39 \%)$ & 0.0004620034 & ko04612 \\
\hline 7 & Protein processing in endoplasmic reticulum & $9(7.6 \%) / 455(1.57 \%)$ & 0.001166426 & ko04141 \\
\hline 8 & Glycosphingolipid biosynthesis - lacto and neolacto series & $4(3.4 \%) / 94(0.33 \%)$ & 0.002007803 & ko00601 \\
\hline 9 & Thyroid hormone signaling pathway & $11(9.2 \%) / 746(2.58 \%)$ & 0.003509184 & ko04919 \\
\hline 10 & AMPK signaling pathway & $8(6.7 \%) / 465(1.61 \%)$ & 0.005048467 & ko04152 \\
\hline 11 & Nitrogen metabolism & $2(1.7 \%) / 19(0.07 \%)$ & 0.005079646 & ko00910 \\
\hline 12 & Vibrio cholerae infection & $14(11.8 \%) / 1170$ (4.05\%) & 0.006652947 & ko05110 \\
\hline 13 & Mineral absorption & $5(4.2 \%) / 214(0.74 \%)$ & 0.007464861 & ko04978 \\
\hline 14 & Adipocytokine signaling pathway & $4(3.4 \%) / 153(0.53 \%)$ & 0.01119608 & ko04920 \\
\hline 15 & ECM-receptor interaction & $7(5.9 \%) / 440(1.52 \%)$ & 0.01272333 & ko04512 \\
\hline 16 & Toxoplasmosis & 4 (3.4\%) / $172(0.6 \%)$ & 0.01655378 & ko05145 \\
\hline 17 & Insulin secretion & $5(4.2 \%) / 264(0.91 \%)$ & 0.01723485 & ko04911 \\
\hline 18 & Thyroid hormone synthesis & $4(3.4 \%) / 195(0.67 \%)$ & 0.02489002 & ko04918 \\
\hline 19 & PI3K-Akt signaling pathway & $14(11.8 \%) / 1386$ (4.8\%) & 0.0255091 & ko04151 \\
\hline 20 & Insulin resistance & 4 (3.4\%) /214 (0.74\%) & 0.0333897 & ko04931 \\
\hline
\end{tabular}

(ko00220) (Additional file 1: Table S8), alanine, aspartate and glutamate metabolism (ko00250) (Additional file 1: Table S8), lysine degradation (ko00310) (Additional file 1: Table S8), valine, leucine and isoleucine degradation (ko00280) (Additional file 1: Table S8), amino sugar and nucleotide sugar metabolism (ko00520) (Additional file 1: Table S8), biosynthesis of amino acids (ko01230) (Additional file 1: Table S8), pyrimidine metabolism (ko00240) (Additional file 1: Table S8). In addition, several important signal pathways were found, cAMP signaling pathway (ko04024) (Additional file 1: Table S6), MAPK signaling pathway (ko04013, ko04010) (Additional file 1: Table S6), Wnt signaling pathway (ko04310) (Additional file 1: Table S6), mTOR signaling pathway (ko04150) (Additional file 1: Table S6), Ras signaling pathway (ko04014) (Additional file 1: Table S6). The results implied these pathways might all take part in osmoregulation.

\section{Validity of DEGs in transcriptomic data}

Ten differentially expressed genes were randomly sampled for verification by transcriptional level experiments, and the results were consistent with those of DEG analyses (Table 4 \& Additional file 1: Figure S3), indicating that the results of DEG were reliable.

\section{Discussion}

The mechanism of osmotic regulation of aquatic crustaceans has received attention in recent years. Extensive work and quite a few important results have been reported on the morphological structure of osmotic regulatory organs [5, 24], ion transport regulation [10, 23, 33], regulation of hemolymph osmoregulation ([19];Huong et al.,

Table 4 Validity of DEGs in Transcriptomic data

\begin{tabular}{lll}
\hline Gene ID & Transcriptome results & verification results \\
\hline Gene.7653 & up & up \\
Gene.16162 & up & up \\
Gene.54888 & up & up \\
Gene. 22643 & up & up \\
Gene.6925 & up & up \\
Gene. 803 & down & down \\
Gene. 18,132 & down & down \\
Gene. 1196 & down & down \\
Gene. 397 & down & down \\
Gene. 2748 & down & down
\end{tabular}

Gene. 7653: CL1096.Contig1_All, Gene. 16,162: CL2951.Contig2_All, Gene. 54,888: Unigene41750_All, Gene. 22,643: CL4861.Contig2_All, Gene. 6925: CL979.Contig3_All, Gene. 803: CL94.Contig5_All, Gene. 18,132: CL3482.Contig1_All, Gene. 1196:CL4395.Contig1_All, Gene. 397: CL41.Contig2_All, Gene. 2748: CL358.Contig1_All. The S. paramamosain beta-actin gene and 18S rRNA gene were selected as the internal control 
2001, [42]), and neuroendocrine regulation [13, 29]. For both euryhaline and stenohaline species, changes in salinity will result in the organism adapting through the regulation of the neuroendocrine system, osmotic regulatory organs (mainly gills), hematopoietic osmotic pressure, and ion transport. A series of changes will occur to adapt to the changing external environment in order to maintain normal physiological and metabolic activity. However, few studies on neuroendocrine regulation, regulation of ion transport enzymes, or osmotic regulation are reported for S. paramamosain. Thus, this paper focuses on osmotic regulation in aquatic crustaceans. Since sequencing of the genome of S. paramamosain has not been completed yet, much of the information obtained still depends on transcriptomics.

S. paramamosain is a euryhaline species, and especially loves living in shallow sea and estuary nearshore. In China, the salinity of seawater in $S$. paramamosain ponds on the farm is between $25 \%$ o and $3 \%$ in most area, and the minority such as in Shanghai is below $3 \%$. There is a production experience in the actual production process that amplitude of variation in salinity exceeding $10 \%$ would cause death for mud crabs (This only refers to sudden salinity drop). $23 \%$ is a normal salinity of the seawater for juvenile crabs S. paramamosain, which was living in the salinity before our treatment. We had made a preliminary experiment to select a salinity for treatment with 10 juvenile crabs as a group, including $13 \%$, $8 \%$, $5 \%$, $3 \%$, and $1 \%$, and the degree of salinity drop was $10 \%, 15 \%, 18 \%, 20 \%$, and $22 \%$, respectively. In the end, we found some individuals in salinity $3 \%$ begin to die, and most individuals nearly died in salinity $1 \%$. So $3 \%$ might be the optimal choice as a critical point for the research of adaptive mechanism responding to sudden drop in salinity, and we finally selected the $3 \%$ in our study. Moreover, the LS death time was concentrated within 24, 48 , and $72 \mathrm{~h}$. The LS group showed hyperactivity within $48 \mathrm{~h}$, and as time went by, the motility was diminished and normalized. The LS group did not have food over $72 \mathrm{~h}$, and gradually started to eat over time. Conditions returned to normal after $120 \mathrm{~h}$. To be honest, the mechanism of adaptive process is very complex, and need more in-depth research in the future work. The study aimed at the adaptive mechanism in response to sudden salinity drop. So we compared the CK with $120 \mathrm{~h}$ _group (a state of complete adaptation) to discover the difference of between normal condition and the adaptive status after sudden salinity drop from $23 \%$ o to $3 \%$, and eventually reveal the adaptive mechanisms in response to sudden salinity drop in the mud crab, S. paramamosain at the level of transcription.
To date, reports on the regulation of infiltration of $S$. paramamosain are still quite limited to cloning and expression of the $\mathrm{Na}^{+} / \mathrm{K}^{+}$-ATPase [8] and cloning and expression of glutamate dehydrogenase (GDH) [25]. GHD is an important enzyme for the metabolism of glycine, proline, and alanine, which serve as general osmolytes in aquatic animals. In this study, GO annotation of DEG analysis showed that the most involved genes were derived from the category of catalytic activity of molecular function (Fig. 4 ), suggesting that the possible role of free amino acids in osmotic regulation is by enzymolysis. In addition, we found that $\mathrm{Na}^{+} / \mathrm{K}^{+}$-ATPase is very active through the KEGG pathway classification and functional enrichment of DEGs (Figs. 5 and 6). The results showed that the $\mathrm{Na}^{+} / \mathrm{K}^{+}$-ATPase strengthened the ion exchange function necessary to maintain the osmotic balance required for normal survival after salinity drop from $23 \%$ to $3 \%$. In addition, there were many other KEGG pathways and differentially expressed genes, which might directly or indirectly participate in the regulation of osmotic adjustment of S. paramamosain, providing a valuable data source for subsequent studies.

S. paramamosain normally lives in estuary areas, where the environment is significantly different from freshwater, brackish water and seawater. Sometimes the salinity changes constantly, and this change requires a response in behavior, morphology, and biochemical physiology. In production, the sudden drop in salinity usually results from heavy rainfall in strong convective weather. For example, two typhoons hit Zhuhai in August 2017 in one week in Guangdong Province, causing huge losses to the crab farming industry. In this study, the cumulative salvage rate in six days was $16 \%$ $(24 / 150)$ in the salinity sag test but may be higher in actual production. Because of the more complicated water environment system in production, the sudden drop in salinity is often accompanied by a decrease in water temperature. The comprehensive factors led to a decrease in crab immunity. As a result, there was an increase in pathogenic microorganisms in the water and an increase in crab mortality. In this study, only the salinity was changed and the rest of the environmental factors were controlled. For the first time, the molecular mechanism of $S$. paramamosain adapting to the salinity drop was studied. Through the research presented here, we had discovered a large number of potential genes that are related to the salinity adaptation in S. paramamosain. The possible KEGG pathway provided a basis for further research. In addition, this study was an important supplement to the physiological study of aquatic crustacean infiltration, but also provided a scientific basis for the regulation of crustacean aquaculture. 


\section{Conclusions}

In conclusion, we analyzed transcriptomic changes in the gills after a sudden drop in salinity in S. paramamosain. One hundred thirty-five DEGs annotated by $\mathrm{Nr}$ were screened, of which 108 were up-regulated and 27 were down-regulated. GO analysis showed that catalytic activity (14) had the most participating genes in the 24 s-tier GO terms, indicating that intracellular metabolic activities in S. paramamosain were enhanced. Based on KEGG pathway and biological functional enrichment on DEGs, the top 20 pathways showed that seven pathways were directly related to the active regulation of the $\mathrm{Na}^{+} / \mathrm{K}^{+} \mathrm{ATP}$ enzyme: Proximal tubule bicarbonate reclamation (ko04964), protein digestion and absorption (ko04974), bile secretion (ko04976), thyroid hormone signaling pathway (ko04919), mineral absorption (ko04978), Insulin secretion (ko04911), thyroid hormone synthesis (ko0491), and the regulatory genes in $\mathrm{Na}^{+} / \mathrm{K}^{+}$ATPase were all up-regulated. Additionally, several amino acid metabolism pathways were detected: arginine biosynthesis (ko00220), alanine, aspartate and glutamate metabolism (ko00250), lysine degradation (ko00310), valine, leucine and isoleucine degradation (ko00280), amino sugar and nucleotide sugar metabolism (ko00520), biosynthesis of amino acids (ko01230), pyrimidine metabolism (ko00240). In addition, some famous signal pathways were found, such as cAMP signaling pathway (ko04024), MAPK signaling pathway (ko04013, ko04010), Wnt signaling pathway (ko04310), mTOR signaling pathway (ko04150), Ras signaling pathway (ko04014). Our findings suggest that not only $\mathrm{Na}^{+} / \mathrm{K}^{+}$ATPase and amino acids played a key role in osmoregulation, but also some important signal pathways participated in osmoregulation. Ultimately, survival of $S$. paramamosain may be sustained in new surroundings with a sudden drop in salinity (23\%o fell to $3 \%$ ), by adjusting to the low salinity. The functional genomic studies of DEGs obtained in this study allow for a better understanding of various physiological responses in marine crustaceans induced by a sudden drop in salinity.

\section{Additional file}

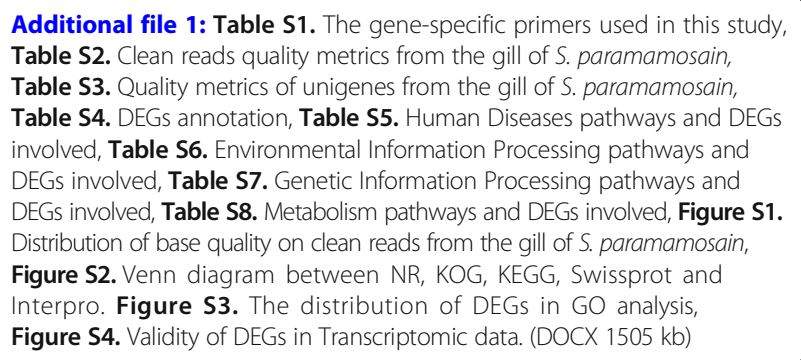

\section{Abbreviations}

GO: Gene Ontology; KEGG: Kyoto Encyclopedia of Genes and Genomes;

LS: Low salinity; S. paramamosain: Scylla paramamosain; sqRT-PCR: semiquantitative reverse-transcription $\mathrm{PCR}$

\section{Acknowledgments}

Aside from funding support, we also thank BGl (BGl, Shenzhen, China) for sequencing consultation and support.

\section{Funding}

This work was supported by the Major Sci \& Tech Special Project of Zhejiang Province (no: 2016C02055-8), Ministry of Agriculture of China \& China Agriculture Research System (no: CARS-48), the K. C. Wong Magna Fund in Ningbo University. The funders had no role in study design, data collection and analysis, decision to publish, or preparation of the manuscript.

\section{Availability of data and materials}

Raw Illumina sequences were deposited in the National Center for Biotechnology Information (NCBI) and our SRA records will be accessible with the following link after the indicated release date: https://www.ncbi.nlm.nih.gov/sra/SRP129841, SRA accession: SRP129841; Temporary Submission ID: SUB3501735.

\section{Authors' contributions}

CW conceived and designed the study. HW and $J$ performed the cultivation of experimental animals, HW, JL, LT, HW, and CM performed and analyzed all the other experiments. HW wrote the manuscript with support from all authors. All authors read and approved the final manuscript.

\section{Ethics approval}

The animal subjects used in the present study are crabs, which are invertebrates and are exempt from this requirement.

\section{Competing interests}

The authors declare that they have no competing interests.

\section{Publisher's Note}

Springer Nature remains neutral with regard to jurisdictional claims in published maps and institutional affiliations.

Received: 9 January 2018 Accepted: 18 May 2018

Published online: 31 May 2018

\section{References}

1. Abe H, Okuma $E$, Amano $H$, et al. Role of free $d$ - and l-alanine in the Japanese mitten crab Eriocheir japonicus to intracellular osmoregulation during downstream spawning migration. Comp Biochem Phys A. 1999; 123(1):55-9.

2. Ahearn GA, Duerr JM, Zhuang Z, et al. lon transport processes of crustacean epithelial cells. Physiol Biochem Zool. 1999;72(1):1-18.

3. Anger K. Salinity tolerance of the larvae and first juveniles of a semiterrestrial grapsid crab, Armases miersii (Rathbun). J Exp Mar Bio Ecol. 1996;202(2):205-23.

4. Anger K. Salinity as a key parameter in the larval biology of decapod crustaceans. Invertebrate reproduction \& development. 2003;43(1):29-45.

5. Barra JA, Pequeux A, Humbert W. A morphological study on gills of a crab acclimated to fresh water. Tissue Cell. 1983;15(4):583-96.

6. Bas C, Spivak E. Effect of salinity on embryos of two southwestern Atlantic estuarine Grapsid crab species cultured in vitro. J Crustacean Biol. 2000;20: 647-56.

7. Charmantier G, Soyez C, Aquacop. Effect of molt stage and hypoxia on osmoregulatory capacity in the peneid shrimp Penaeus vannamei. J Exp Mar Bio Ecol. 1994;178(2):233-46.

8. Chung KF, Lin HC. Osmoregulation and $\mathrm{Na}$, K-ATPase expression in osmoregulatory organs of Scylla paramamosain. Comp Biochem Physiol A Mol Integr Physiol. 2006;144(1):48-57.

9. Conesa A, Götz S, García-Gómez JM, et al. Blast2GO: a universal tool for annotation, visualization and analysis in functional genomics research. Bioinformatics. 2005:21(18):3674-6.

10. Corotto FS, Holliday CW. Branchial Na, K-ATPase and osmoregulation in the purple shore crab, Hemigrapsus nudus (Dana). Comp Biochem Phys A. 1996; 113(4):361-8. 
11. Crambert G, Hasler U, Beggah AT, et al. Transport and pharmacological properties of nine different human Na, K-ATPase isozymes. J Biol Chem. 2000;275(3):1976-86

12. Felder DL. Osmotic and ionic regulation in several western Atlantic callianassidae (crustacea, decapoda, thalassinidea). Biol Bull. 1978;154(3):409-29.

13. Freire CA, McNamara JC, Rosa JC, et al. Neuroendocrine control of osmotic regulation in the freshwater shrimp Macrobrachium olfersii (Wiegmann) (Crustacea, decapoda): free amino acid concentrations in the hemolymph. Gen Comp Endocrinol. 1995;100(1):83-91.

14. Giménez L, Anger K. Relationships among salinity, egg size, embryonic development, and larval biomass in the estuarine crab Chasmagnathus granulata Dana, 1851. J Exp Mar Bio Ecol. 2001;260(2):241-57.

15. Giménez L, Torres G. Larval growth in the estuarine crab Chasmagnathus granulata : the importance of salinity experienced during embryonic development, and the initial larval biomass. Mar Biol. 2002:141:877-85.

16. González-Ortegón E, Pascual E, Cuesta JA, et al. Field distribution and osmoregulatory capacity of shrimps in a temperate European estuary (SW Spain). Estuar Coast Shelf S. 2006;67(1-2):293-302

17. Götz S, García-Gómez JM, Terol J, et al. High-throughput functional annotation and data mining with the Blast2GO suite. Nucleic Acids Res. 2008;36(10):3420-35.

18. Grabherr MG, Haas BJ, Yassour M, et al. Full-length transcriptome assembly from RNA-Seq data without a reference genome. Nat Biotechnol, 2011. 2011;29(7):644-52.

19. Huong DT, Yang WJ, Okuno A, et al. Changes in free amino acids in the hemolymph of giant freshwater prawn Macrobrachium rosenbergii exposed to varying salinities: relationship to osmoregulatory ability. Comp Biochem Physiol A Mol Integr Physiol. 2001;128(2):317-26.

20. Khodabandeh S, Charmantier G, Charmantierdaures M. Ultrastructural studies and $\mathrm{Na}^{+}, \mathrm{K}^{+}$-ATPase immunolocalization in the antennal urinary glands of the lobster Homarus gammarus (Crustacea, Decapoda). J Histochem Cytochem. 2005;53(10):1203-14.

21. Li E, Wang $X$, Chen $K$, et al. Physiological change and nutritional requirement of Pacific white shrimp Litopenaeus vannamei at low salinity. Rev Aquacult. 2017:9:57-75.

22. Lignot JH, Susanto GN, Charmantier-Daures $M$, et al. Immunolocalization of $\mathrm{Na}^{+}, \mathrm{K}^{+}$-ATPase in the branchial cavity during the early development of the crayfish Astacus leptodactylus (Crustacea, Decapoda). Cell Tissue Res. 2005; 319(2):331-9.

23. Limab AG, McNamara JC, Terrac WR. Regulation of hemolymph osmolytes and gill $\mathrm{Na}^{+} / \mathrm{K}^{+}$-ATPase activities during acclimation to saline media in the freshwater shrimp Macrobrachium olfersii (Wiegmann, 1836) (Decapoda, Palaemonidae). J Exp Mar Bio Ecol. 1997;215(1):81-91.

24. Lin SC, Liou CH, Cheng JH. The role of the antennal glands in ion and body volume regulation of cannulated Penaeus monodon reared in various salinity conditions. Comp Biochem Physiol A Mol Integr Physiol. 2000;127(2):121-9.

25. Lu JY, Shu MA, Xu BP, et al. Mud crab Scylla paramamosain glutamate dehydrogenase: molecular cloning, tissue expression and response to hyposmotic stress. Fish Sci. 2015;81:175-86.

26. Lucena MN, Garçon DP, Fontes CF, et al. Polyamines regulate phosphorylationdephosphorylation kinetics in a crustacean gill $\mathrm{Na}^{+}, \mathrm{K}^{+}$-ATPase. Mol Cell Biochem. 2017:429(1-2):187-98.

27. Lucu C, Towle DW. Na ${ }^{+} / \mathrm{K}^{+}$-ATPase in gills of aquatic crustacea. Comp Biochem Physiol A Mol Integr Physiol. 2003;135(2):195-214.

28. Mauro NA. Variations in osmoregulatory capacity in two species of intertida sabellariids (Annelida: Polychaeta) from tropical and mediterranean habitats. Comp Biochem Phys A. 1977;56(3):375-7.

29. Morris S. Neuroendocrine regulation of osmoregulation and the evolution of air-breathing in decapod crustaceans. J Exp Biol. 2001;204:979-89.

30. Pan LQ, Zhang $L$, Liu HY. Effects of salinity and $\mathrm{pH}$ on ion-transport enzyme activities, survival and growth of Litopenaeus vannamei postlarvae. Aquaculture. 2007;273(4):711-20.

31. Pertea $G$, Huang $X$, Liang $F$, et al. TIGR gene indices clustering tools (TGICL): a software system for fast clustering of large EST datasets. Bioinformatics. 2003;19(5):651-2

32. Rahmi N, Zeng C. Effects of temperature and salinity on the survival and development of mud crab, Scylla serrata (Forsskal), larvae. Aquac Res. 2007; 38(14):1529-38.

33. Romano $\mathrm{N}$, Zeng C. Importance of balanced $\mathrm{Na}^{+} / \mathrm{K}^{+}$ratios for blue swimmer crabs, Portunus pelagicus, to cope with elevated ammonia-N and differences between in vitro and in vivo gill $\mathrm{Na}^{+} / \mathrm{K}^{+}$-ATPase responses. Aquaculture. 2011; 318(1-2):154-61.

34. Romano N, Zeng C. Osmoregulation in decapod crustaceans: implications to aquaculture productivity, methods for potential improvement and interactions with elevated ammonia exposure. Aquaculture. 2012;334337(1):12-23.

35. Ruscoe IM, Shelley CC, Williams GR. The combined effects of temperature and salinity on growth and survival of juvenile mud crabs (Scylla serrata Forskal). Aquaculture. 2004;238(1-4):239-47.

36. Sabat $\mathrm{P}$, Maldonado K, Fariña JM, et al. Osmoregulatory capacity and the ability to use marine food sources in two coastal songbirds (Cinclodes: Furnariidae) along a latitudinal gradient. Oecologia. 2006;148(2):250-7.

37. Sakuragui MM, Sanches JR, Fernandes MN. Gill chloride cell proliferation and respiratory responses to hypoxia of the neotropical erythrinid fish Hoplias malabaricus. J Comp Physiol B. 2003:173(4):309-17.

38. Tantulo U, Fotedar R. Comparison of growth, osmoregulatory capacity, ionic regulation and organosomatic indices of black tiger prawn (Penaeus monodon Fabricius, 1798) juveniles reared in potassium fortified inland saline water and ocean water at different salinities. Aquaculture. 2006;258(1-4):594-605.

39. Tucker RK, Costlow JD Jr. Free amino acid changes in normal and eyestalkless megalopa larvae of the blue crab, Callinectes sapidus, during the course of the molt cycle. Comp Biochem Physiol A Comp Physiol. 1975:51(1A):75-8.

40. Wang GZ, Lin SJ, Lin QW, et al. Effects of salinity on survival and development of the mud crab scylla serrata. J Fish China. 1998;22(1):89-92. in Chinese

41. Wang $H$, Wang $L$, Wang $Y$, et al. High yield exogenous protein HPL production in the Bombyx mori silk gland provides novel insight into recombinant expression systems. Sci Rep. 2015;5:13839.

42. Wang WN, Wang AL, Bao $L$, et al. Changes of protein-bound and free amino acids in the muscle of the freshwater prawn Macrobrachium nipponense in different salinities. Aquaculture. 2004;233(1):561-71.

43. Weber RE, Van Marrewijk WJA. Free amino acids in the shrimp crangon crangon and their osmoregulatory significance. Neth J Sea Res. 1972; 5(4):391-415.

44. Wheatly MG, Zanotto FP, Hubbard MG. Calcium homeostasis in crustaceans: subcellular ca dynamics. Comp Biochem Physiol B Biochem Mol Biol. 2002; 132(1):163-78

45. Wilder MN, Ikuta K, Atmomarsono M, et al. Changes in osmotic and ionic concentrations in the hemolymph of Macrobrachium rosenbergii, exposed to varying salinities and correlation to ionic and crystalline composition of the cuticle. Comp Biochem Phys A. 1998;119(4):941-50.

46. Xu DF, Liu ZH, Xu XH, et al. The influence of typhoon on the sea surface salinity in the warm pool of the western Pacific. Acta Oceanol Sin. 2005; 27(6):9-15. in Chinese

47. Xu Q, Liu Y. Gene expression profiles of the swimming crab Portunus trituberculatus, exposed to salinity stress. Mar Biol. 2011;158(10):2161-72.

Ready to submit your research? Choose BMC and benefit from:

- fast, convenient online submission

- thorough peer review by experienced researchers in your field

- rapid publication on acceptance

- support for research data, including large and complex data types

- gold Open Access which fosters wider collaboration and increased citations

- maximum visibility for your research: over $100 \mathrm{M}$ website views per year

At BMC, research is always in progress.

Learn more biomedcentral.com/submissions 